Delft University of Technology

\title{
Long-Lived Solid-State Optical Memory for High-Rate Quantum Repeaters
}

Askarani, Mohsen Falamarzi; Das, Antariksha; Davidson, Jacob H.; Amaral, Gustavo C.; Slater, Joshua A.; Marzban, Sara; Cone, Rufus L.; Oblak, Daniel; Tittel, Wolfgang; More Authors

DOI

10.1103/PhysRevLett.127.220502

Publication date

2021

Document Version

Final published version

Published in

Physical Review Letters

\section{Citation (APA)}

Askarani, M. F., Das, A., Davidson, J. H., Amaral, G. C., Slater, J. A., Marzban, S., Cone, R. L., Oblak, D., Tittel, W., \& More Authors (2021). Long-Lived Solid-State Optical Memory for High-Rate Quantum

Repeaters. Physical Review Letters, 127(22), [220502]. https://doi.org/10.1103/PhysRevLett.127.220502

\section{Important note}

To cite this publication, please use the final published version (if applicable).

Please check the document version above.

\section{Copyright}

Other than for strictly personal use, it is not permitted to download, forward or distribute the text or part of it, without the consent of the author(s) and/or copyright holder(s), unless the work is under an open content license such as Creative Commons.

Takedown policy

Please contact us and provide details if you believe this document breaches copyrights.

We will remove access to the work immediately and investigate your claim. 


\title{
Long-Lived Solid-State Optical Memory for High-Rate Quantum Repeaters
}

\author{
Mohsen Falamarzi Askarani®, ${ }^{1, *}$ Antariksha Das $\odot,{ }^{1, *}$ Jacob H. Davidson, ${ }^{1}$ Gustavo C. Amaral $\odot,{ }^{1}$ Neil Sinclair, ${ }^{2,3}$ \\ Joshua A. Slater, ${ }^{1}$ Sara Marzban, ${ }^{1}$ Charles W. Thiel, ${ }^{4}$ Rufus L. Cone, ${ }^{4}$ Daniel Oblak $\odot,{ }^{5}$ and Wolfgang Tittel ${ }^{1,6,7}$ \\ ${ }^{1}$ QuTech and Kavli Institute of Nanoscience, Delft University of Technology, 2628 CJ Delft, Netherlands \\ ${ }^{2}$ John A. Paulson School of Engineering and Applied Sciences, Harvard University, Cambridge, Massachusetts 02138, USA \\ ${ }^{3}$ Division of Physics, Mathematics and Astronomy, and Alliance for Quantum Technologies (AQT), California Institute of Technology, \\ Pasadena, California 91125, USA \\ ${ }^{4}$ Department of Physics, Montana State University, Bozeman, Montana 59717, USA \\ ${ }^{5}$ Institute for Quantum Science and Technology, and Department of Physics \& Astronomy, University of Calgary, \\ Calgary, Alberta T2N 1N4, Canada \\ ${ }^{6}$ Department of Applied Physics, University of Geneva, 1211 Geneva 4, Switzerland \\ ${ }^{7}$ Schaffhausen Institute of Technology - SIT, 1211 Geneva 4, Switzerland
}

(Received 4 June 2021; accepted 20 October 2021; published 22 November 2021)

\begin{abstract}
We argue that long optical storage times are required to establish entanglement at high rates over large distances using memory-based quantum repeaters. Triggered by this conclusion, we investigate the $795.325 \mathrm{~nm}^{3} \mathrm{H}_{6} \leftrightarrow{ }^{3} \mathrm{H}_{4}$ transition of Tm: $\mathrm{Y}_{3} \mathrm{Ga}_{5} \mathrm{O}_{12}$ (Tm:YGG). Most importantly, we find that the optical coherence time can reach $1.1 \mathrm{~ms}$, and, using laser pulses, we demonstrate optical storage based on the atomic frequency comb protocol during up to $100 \mu \mathrm{s}$ as well as a memory decay time $T_{m}$ of $13.1 \mu \mathrm{s}$. Possibilities of how to narrow the gap between the measured value of $T_{m}$ and its maximum of $275 \mu$ s are discussed. In addition, we demonstrate multiplexed storage, including with feed-forward selection, shifting and filtering of spectral modes, as well as quantum state storage using members of nonclassical photon pairs. Our results show the potential of Tm:YGG for creating multiplexed quantum memories with long optical storage times, and open the path to repeater-based quantum networks with high entanglement distribution rates.
\end{abstract}

DOI: 10.1103/PhysRevLett.127.220502

The future quantum internet $[1,2]$ will enable one to share entanglement and hence quantum information over large distances-ultimately between any two points on Earth. To overcome attenuation in optical fibers, quantum repeaters are needed [3-7], many of which require quantum memories for light [8]. Such memories allow storing qubits, encoded into photons that have traveled over long distances, until feed-forward information becomes available. This information specifies which optical mode-including spectral, spatial, and temporal modes-a qubit should occupy once it has been reemitted from the memory. Note that the required mode assignment (or mode mapping operation) can happen in a memory-internal manner, e.g., by controlling when a photon is reemitted (also known as read-out on demand) [4,9-11], or externally, e.g., by directing the emitted photon to a specific spatial mode [12] or by shifting its spectrum [6].

To maximize the entanglement distribution rate of a quantum repeater, qubits must be added continuously to the memory - not only once a previously stored qubit has been reemitted but also while it is still being stored. Such multiplexed storage implies (a) the use of large ensembles of absorbers that enable bipartite entanglement with many photonic qubits; and (b) that any memory-specific control operation, triggered by the absorption of a newly arriving qubit, must neither affect reemission nor the possibility for mode mapping of a previously absorbed qubit. Stated differently, any control operation required after absorption of a qubit or a train of qubits must not introduce dead time that prevents the memory from accepting additional qubits. This would cause a reduction of the memory's timebandwidth product $[13,14]$ and, when used as an element of a quantum repeater, a reduction in the entanglement distribution rate (see Supplemental Material [15] for an example).

Unfortunately, the latter requirement of qubit independence (b) can be at odds with a high repetition rate. As we show below, one example is that of temporal multiplexing and read-out on demand in the so-called atomic frequency comb (AFC) quantum memory protocol, which requires one to temporarily map qubit states between optical and spin coherence [16]. This leads us to conclude that it is important to optimize the optical storage time, i.e., the time during which qubits are stored as optical coherence, which can be excited using visible or near infrared light. It is important to realize that this conclusion also holds in the case of purely optical storage (no spin mapping), and regardless of the degree of freedom used for multiplexing. 


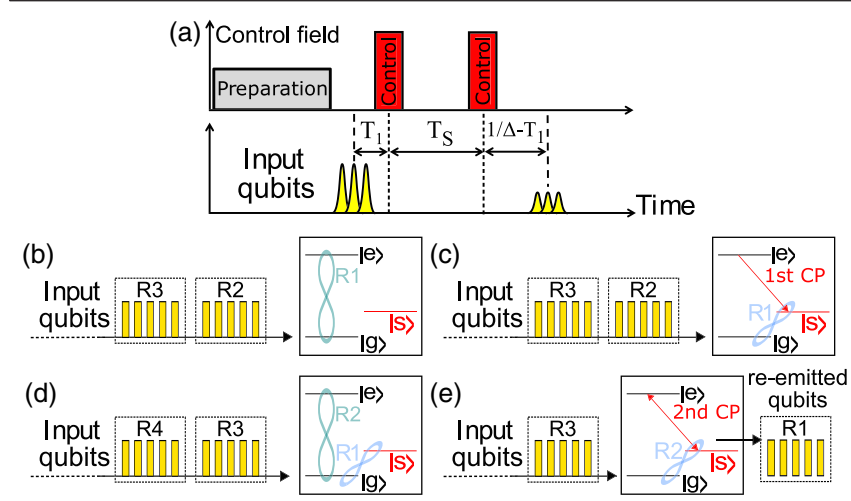

FIG. 1. Recall on demand using the AFC spin-wave storage protocol. (a) Pulse sequence. (b)-(e) Memory input and output, as well as atomic coherence (indicated by light blue and green figures of eight) for different moments during storage (see text for details). Trains of qubits are labeled $R 1-R 4$. CP: Control pulse. A three-level lambda system is formed by spin states $|g\rangle$ and $|s\rangle$, and by excited state $|e\rangle$.

Triggered by this finding, we investigate thulium-doped yttrium gallium garnet $\left(\mathrm{Tm}^{3+}: \mathrm{Y}_{3} \mathrm{Ga}_{5} \mathrm{O}_{12}\right.$ or Tm:YGG)-a rare-earth-ion doped crystal (REIC) whose promising spectroscopic properties have been demonstrated previously $[17,18]$, but whose potential for storing photonic qubits has not yet been established. Here, we show that its optical coherence time $T_{2}$ can reach $1.1 \mathrm{~ms}$, which is one of the longest times reported for any REIC [19,20]. Motivated by this promising result, we investigate Tm:YGG for AFCbased memory, and demonstrate optical storage of laser pulses up to $100 \mu \mathrm{s}$. This is comparable with recent results obtained using $\mathrm{Yb}: \mathrm{Y}_{2} \mathrm{SiO}_{5}$ [21] and $\mathrm{Eu}: \mathrm{Y}_{2} \mathrm{SiO}_{5}$ [22], and exceeds all other reported results of storage of light in optical coherence with any REIC by at least a factor of 10 . However, we also find that the memory decay time $T_{m}$ of around $13 \mu \mathrm{s}$ is 20 times smaller than the $T_{2}$-imposed maximum of $275 \mu \mathrm{s}$. Before addressing reasons for this large gap, we confirm the possibility for spectrally multiplexed storage and feed-forward-based spectral mode mapping [6], which allows using memory materialsincluding Tm-doped crystals-whose atomic level structure lacks the spin states required for memory-internal temporal mode mapping. Furthermore, we also show that quantum correlations between members of photon pairs persist throughout storage, i.e., that our memory can operate in the quantum regime. We conclude by mentioning reasons for the currently small memory efficiency, leaving more details to Supplemental Material [15] which includes Refs. [23,24].

The need for long optical storage.-To support our claim that qubit independence can be at odds with a high repetition rate, let us discuss the example of temporal mode mapping using the AFC spin-wave memory in REICs [11]. As depicted in Fig. 1(a), a pair of optical control pulses ( $\pi$ pulses that resonantly couple the excited state $|e\rangle$ with a ground state $|s\rangle$ ) allows one to reversibly map optical coherence onto a spin transition. In this case, the timing of the second control pulse determines when the photons will be reemitted from the memory, allowing for readout on demand.

Let us now assume that a first train, $R 1$, of temporally multiplexed qubits has already been absorbed by the memory, that the first control pulse has been applied, and that a second train, $R 2$, of qubits has just been added to the memory [Figs. 1(b)-1(d)]. At this point, $R 1$ is stored in terms of spin coherence, and $R 2$ in terms of optical coherence. Unfortunately, the subsequent control pulse, applied to the memory with the goal to map $R 2$ onto spin coherence, simultaneously maps $R 1$ back onto optical coherence. This causes reemission of these qubits at a time that is determined by the need to transfer the second train, rather than by feed-forward information that specifies what to do with the first.

Fortunately, this problem can be avoided by storing only one train at a time. But in order to maximize the repetition rate of the repeater (or to minimize the memory's dead time), this block, and hence the time during which qubits are stored in optical coherence, should be as long as possible-ideally as long as the total storage time. At the same time, long optical storage times allow maximizing the elementary link length in quantum repeater architectures that do not employ mapping between optical and spin coherence. In turn, this leads to higher entanglement distribution rates as it reduces the number of (currently inefficient) Bell-state measurements that are required to connect neighboring links [6].

Tm:YGG and experimental setup.-Because of their unique spectroscopic properties [25], REICs have been broadly explored over the last two decades for quantum technology [26,27], e.g., as ensemble-based quantum memory for light $[8,28,29]$ or for quantum processing [30,31]. But while significant effort has been spent to increase storage times in spin coherence [32-35]), much less work has been devoted to advancing and better understanding the limitations of storage in optical coherence. To address this shortcoming, we use a 25-mm-long, 1\% Tm:YGG crystal, mounted inside an adiabatic demagnetization refrigerator cooled to $\sim 500 \mathrm{mK}$. YGG forms a cubic lattice in which $\mathrm{Tm}^{3+}$ replaces $\mathrm{Y}^{3+}$ in six crystallographically equivalent sites of local $D_{2}$ point group symmetry [36]. An ideal Tm:YGG crystal is optically isotropic. Magnetic fields up to $2 \mathrm{~T}$ can be applied along the crystal $\langle 111\rangle$ axis, splitting all electronic levels through the enhanced Zeeman interaction into two hyperfine sublevels $[18,37]$. Figure 2 (inset) depicts a simplified level structure.

We use a tunable continuous-wave diode laser at $795.325 \mathrm{~nm}$ wavelength to address the ${ }^{3} \mathrm{H}_{6} \leftrightarrow{ }^{3} \mathrm{H}_{4}$ zerophonon line. Because of the use of a non-polarizationmaintaining fiber, the polarization state at the input of the crystal is unknown. Furthermore, it evolves inside the 


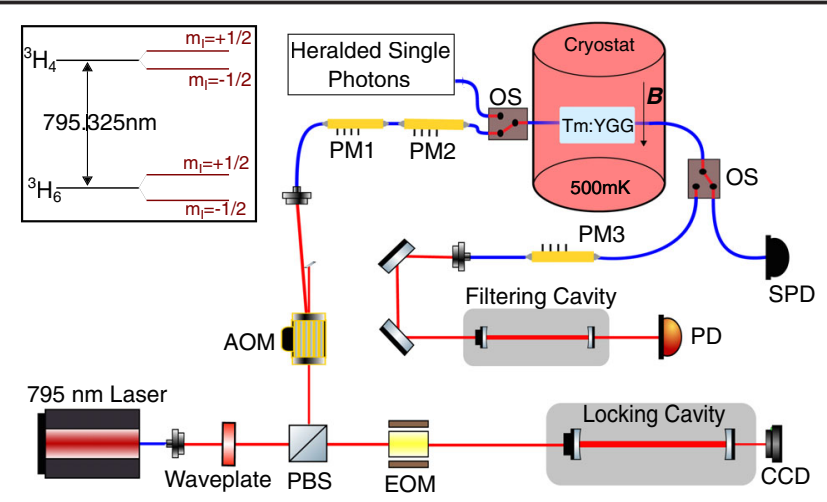

FIG. 2. Experimental setup. AOM: acousto-optic modulator; PM: phase modulator; OS: optical switch; EOM: electro-optic modulator; PD: Classical photo detector; SPD: Single photon detector; CCD: charge-coupled device camera; $\boldsymbol{B}$ : Magnetic field. Inset: simplified energy level diagram of Tm:YGG showing the ${ }^{3} \mathrm{H}_{6} \leftrightarrow{ }^{3} \mathrm{H}_{4}$ zero-phonon line.

crystal due to birefringence stemming from imperfect crystal growth [17]. The laser is frequency locked to a high finesse cavity using the Pound-Drever-Hall method, resulting in an instability over $\sim 100 \mu$ s below $20 \mathrm{kHz}$. To intensity modulate and frequency modulate the light, we use a single-pass acousto-optic modulator (AOM) (driven by a rf signal generator) and a phase modulator (PM) (driven by an arbitrary waveform generator). After passing through the crystal's $\langle 110\rangle$ direction, the light is directed to a photodetector. This setup is used for AFC creation (see also [38]), initial memory characterization, and storage of optical pulses in a single spectral mode. For frequencymultiplexed storage and feed-forward recall, additional phase modulators are used to add frequency sidebands to the laser light, each of which creates a memory in a different spectral segment, and to frequency shift the light after reemission so that only the desired spectral mode passes through an optical filter cavity [6]. In addition to laser pulse, we can also send heralded single photons into the memory, see Fig. 2.

Measurements and results.-First, as a key property that determines the maximum optical storage time, we characterized the optical coherence time $T_{2}$ as a function of magnetic field using two-pulse photon echoes [39]. As an important difference compared to our previous studies $[17,18]$, the temperature was lowered from $1.2 \mathrm{~K}$ to $500 \mathrm{mK}$. As shown in Figs. 3(a) and 3(b) and predicted earlier [17], this resulted in a very significant improvement of the coherence time from $490 \mu \mathrm{s}$ to around $1.1 \mathrm{~ms}$ - one of the longest reported optical coherence times for any rareearth crystal and approaching the limit of $2.6 \mathrm{~ms}$ imposed by the ${ }^{3} \mathrm{H}_{4}$ population lifetime [18].

Next, we investigated the possibility for optical data storage, both using laser pulses as well as quantum states of light. Toward this end, we employed the two-level atomic frequency comb protocol [16]. An AFC is characterized by
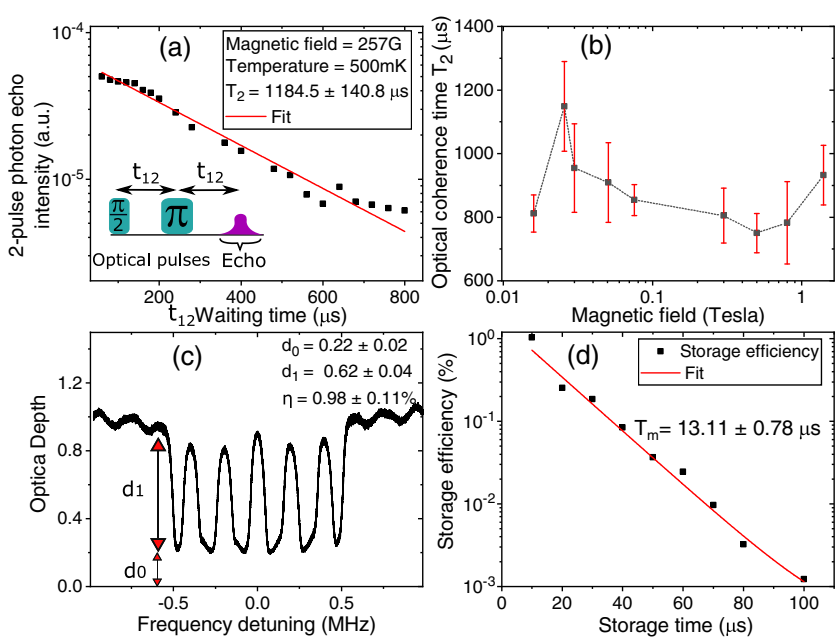

FIG. 3. Optical coherence, and optical data storage in a single spectral mode. (a) Exponential decay of the two-pulse photon echo signal as a function of the delay between the two optical pulses. (b) Optical coherence time $T_{2}$ as a function of magnetic field. The dashed line guides the eye. (c) AFC of $1 \mathrm{MHz}$ bandwidth tailored for $5 \mu \mathrm{s}$ storage time. The calculated efficiency $\eta$ is approximately $1 \%$. (d) Measured memory efficiency as a function of storage time using AFCs with finesse 2. In all measurements $T \sim 500 \mathrm{mk}$. The error bars in (a) and (d) are smaller than the data points.

an absorption profile composed of evenly spaced teeth in the frequency domain, which can be created using frequency-selective optical pumping of population from the troughs of the AFC to other atomic levels. Note that Tm: YGG is well suited for this task due to long-lived hyperfine levels within the electronic ground state manifold [18]. Absorption of a photon with wave number $k$ by an AFC results in the creation of a collective atomic excitation described by $|\psi\rangle_{A}=N^{-1 / 2} \sum_{j=1}^{N} c_{j} e^{i 2 \pi \delta_{j} t} e^{-i k z_{j}}\left|e_{j}\right\rangle$. Here, $N$ is the number of ions in the AFC, $\left|e_{j}\right\rangle$ a state in which only ion $j$ is excited, $\delta_{j}$ the detuning of this ions transition with respect to the input photons carrier frequency, and $z_{j}$ and $c_{j}$ its position and excitation probability amplitude, respectively. After initial dephasing, the coherence rephases, resulting in reemission of the photon after a time $\tau$ that is determined by the inverse AFC tooth spacing $\Delta$, where $\tau=1 / \Delta$. See [16,22] for more details. An example of an Tm:YGG AFC is depicted in Fig. 3(c).

(a) Long-lived storage of laser pulses.-Given the remarkable optical coherence time, it is important to assess how the memory efficiency evolves with storage time. To this end, we used 1- $\mu$ s-long laser pulses (note that the use of true single photons would not change the results), and created AFCs with finesse $F$ - the ratio between AFC peak spacing $\Delta$ and peak width $\delta$-of 2. See [6,38] for more information. Tooth spacings varied between 100 and $10 \mathrm{kHz}$, corresponding to storage times between 10 and $100 \mu \mathrm{s}$, respectively. The choice of $F=2$ maximizes the 

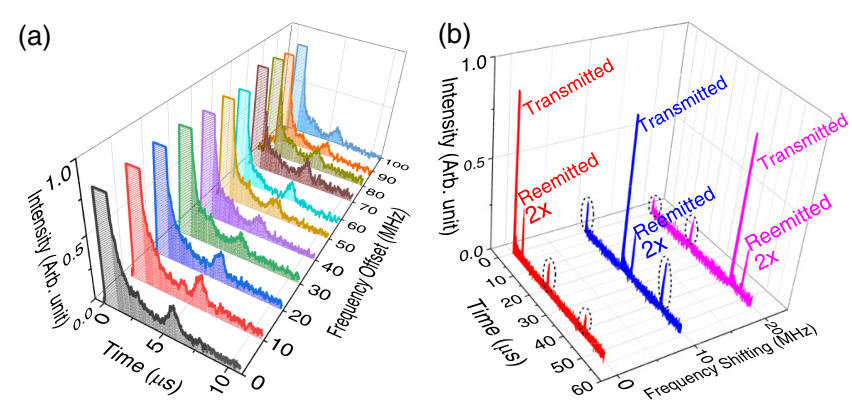

FIG. 4. Storage of data in multiple spectral modes. (a) Spectrally multiplexed AFC quantum memory used to simultaneously store optical pulses in 11 spectral modes for $5 \mu \mathrm{s}$. (b) Feed-forward mapping of spectral modes onto one with zero detuning. Laser pulses in three spectral modes were stored and reemitted after $5 \mu$ s. Frequency shifting using phase modulator PM3 allowed mapping any desired mode onto one with zero detuning. Only this mode was transmitted through the filter cavity. The reemitted pulses are magnified by a factor of 2 , and crosstalk is indicated using dotted circles.

storage efficiency (see Sec. 2B in Supplemental Material [15] which includes Refs. [40,41]), which was limited due to the crystal's small optical depth of around 1. The AFC bandwidth for all storage times was $0.5 \mathrm{MHz}$ except for $100 \mu \mathrm{s}$, where it was reduced to $0.2 \mathrm{MHz}$. Because of the need for highly resolved AFCs with narrow-linewidth teeth, their preparation took $1 \mathrm{~s}$. This time was followed by a waiting time of $20 \mathrm{~ms}$-approximately 15 times the radiative lifetime of the ${ }^{3} \mathrm{H}_{4}$ level-to avoid detecting spurious photons caused by spontaneous decay of ions excited during the AFC preparation. The magnetic field in all measurements was around $100 \mathrm{G}$, which maximizes the optical coherence time.

We detected reemitted pulses after up to $100 \mu \mathrm{s}$ and found that the memory storage efficiency decreases exponentially as a function of storage time with a decay constant $T_{m}=13.1 \pm 0.8 \mu$ s [see Fig. 3(d)]. This value is much smaller than the ultimate limit $T_{m}^{\max }$ imposed by $T_{2}$ of around $275 \mu \mathrm{s}$. Possible reasons are listed in the outlook and detailed Supplemental Material [15] which includes Refs. [21,42-44].

(b) Frequency-multiplexed storage with feed-forward mode mapping.-To demonstrate spectral multiplexing, we prepared 11 AFCs with $F=2$, each of $1 \mathrm{MHz}$ bandwidth and spaced by $10 \mathrm{MHz}$, over a total bandwidth of $100 \mathrm{MHz}$. Laser pulses of $1 \mu$ s duration were created in each spectral mode. They were stored and recalled after $5 \mu \mathrm{s}$ [see Fig. 4(a)]. Note that the individual modes were resolved by changing the resonance frequency of the filtering cavity (see Fig. 2). Assuming that five subsequent pulses-each of $1 \mu \mathrm{s}$ duration-fit into the storage time, this results in a multimode capacity over spectral and temporal degrees of freedom of 55. Note that the storage time-significantly less than our maximum of $100 \mu \mathrm{s}$ in this and all subsequent measurements - was limited by a trade-off between a more complex AFC tailoring procedure and memory efficiency. Otherwise, all parameters used to create the AFCs remained unchanged.

To implement feed-forward mode mapping, imposed by the use of a multiplexed memory in a quantum repeater, we furthermore demonstrated spectral shifting of the recalled laser pulses such that only the desired spectral mode was subsequently transmitted through a filter cavity with a fixed resonance frequency. Our approach, which is further explained in Supplemental Material [15] (which includes Refs. [45-53]) and in [6], is equivalent to the more wellknown case of temporal multiplexing, in which one has to retrieve photons in specific temporal modes. Since the mode mapping in our case is performed in the frequency domain, the storage time of the memory is fixed; it corresponds to the round-trip time from the end of an elementary link to its center and back. The results of our proof-of-principle demonstration are depicted in Fig. 4(b).

For these measurements, the internal storage efficiency (calculated by comparing the energies of input and reemitted pulses and after considering coupling loss) was around $1.3 \%$. Note that number of spectral channels can easily be increased with more laser power, allowing for paralleled AFC generation. Given the inhomogeneous broadening of the ${ }^{3} \mathrm{H}_{6} \leftrightarrow{ }^{3} \mathrm{H}_{4}$ transition of $56 \mathrm{GHz}$, this could in principle yield thousands of spectral modes. Furthermore, increasing the storage time by an order of magnitude—still much less then $T_{m}^{\max }$ — would improve the multimode capacity by another factor of 10 .

(c) Storage of heralded single photons.-Finally, we verified that Tm:YGG, together with the two-level AFC protocol, is suitable for quantum state storage. As described in detail in [48], we created pairs of quantum-correlated photons at 795 and $1532 \mathrm{~nm}$ wavelength by means of spontaneous parametric down-conversion of strong laser pulses in a periodically poled $\mathrm{LiNbO}_{3}$ crystal. The detection of a $1532 \mathrm{~nm}$ photon using a superconducting nanowire single-photon detector heralded the presence of a $795 \mathrm{~nm}$ photon, which was directed into, stored in, and released after $43 \mathrm{~ns}$ from the Tm:YGG memory. Note that the memory creation procedure remained unchanged except that the AFC bandwidth was increased to $4 \mathrm{GHz}$ to better match the photon bandwidth, and that the magnetic field was increased to $3 \mathrm{kG}$ to match the difference in ground and excited state level splitting with the spacing between a trough and the neighboring tooth in the AFC [40]. The latter also increased the persistence of the AFC, allowing us to repeat the preparation sequence only every $10 \mathrm{~s}$. Together with the preparation time of $1 \mathrm{~s}$, this resulted in a memory availability of around $90 \%$. The photons were then detected using a single-photon detector based on a silicon avalanche photodiode. The system efficiency, assessed by comparing photon detection rates with and without memory was $0.05 \%$. Taking $15 \%$ fiber coupling into account, this yields an internal storage efficiency of $0.35 \%$. 
To verify that the nonclassical correlations with the $1532 \mathrm{~nm}$ photons persist throughout the storage process, we measured the second order cross-correlation coefficient $g_{12}^{(2)}(t)$ between the two photons of a pair using timeresolved coincidence detection [38]. Before storage, we found $g_{12}^{(2)}(0 \mathrm{~ns})=18 \pm 0.02$ and, importantly, after $43 \mathrm{~ns}$ storage $g_{12}^{(2)}(43 \mathrm{~ns})=4.58 \pm 0.46$ (this value is reduced due to excess loss combined with detector dark counts). See Supplemental Material [15]. Both values surpass the classical upper bound of 2, confirming the quantum nature of the photon source as well as the memory.

Discussion and conclusion.-Our investigations have resulted in an optical coherence time $T_{2}$ up to $1.1 \mathrm{~ms}$ and optical storage times up to $100 \mu \mathrm{s}$. However, they also revealed a memory decay time of $13.1 \mu \mathrm{s}$ - significantly smaller than the limit imposed by $T_{2}$, and in general a small recall efficiency. To increase the memory performance to a level that allows its use in a quantum network, several improvements, most, if not all of which are of technical nature, are required. As we describe in more detail in Supplemental Material [15], this includes using a frequency-stabilized laser with narrower linewidth [54,55], a cryostat with reduced vibrations [56], a more stable magnetic field as well as finding parameters (propagation direction and polarization of the light [57], and external magnetic field) under which spectral diffusion due to ionion interactions [58] is reduced and the ${ }^{3} \mathrm{H}_{6} \leftrightarrow{ }^{3} \mathrm{H}_{4}$ transition becomes a so-called clock transition [37,59-62]. Furthermore, to counter the effects of limited optical depth, the light-atom interaction has to be enhanced using an impedance-matched cavity [33,63-66]. This also removes the problem of re-absorption of photons that are emitted in forward direction. Furthermore, we can improve the optical pumping by changing the currently sequential excitation of narrow spectral intervals within the inhomogeneously broadened Tm transition by complex hyperbolic secant pulses $[22,67]$. This will lead to better confined teeth with a more squarish spectral profile, and hence to a reduced background within the troughs in between these teeth. At the same time, it will also allow creating AFCs with higher finesse, resulting in reduced decoherence during photon storage $[22,44,68]$.

Finally, note that despite the currently small multiplexing capability -in particular compared to the 1060 temporal modes in [69] — our demonstration shows the advantage of using atomic ensembles over single absorbers where it is limited to one. Note as well that coupling of a single spin, e.g., a diamond nitrogen-vacancy center, to neighboring nuclear spins does not solve this issue due to the limited number of interacting neighbors-e.g., 27 in [70]—and the small coupling strength.

We anticipate that further improvements of our work will lead to long-lived, efficient, and multimode optical quantum memories that enable the efficient distribution of entanglement across extended quantum networks.
The authors thank M. Grimau Puigibert, T. Chakraborty, and O. P. Casas for experimental help and M. Afzelius for discussions. We acknowledge funding through the Netherlands Organization for Scientific Research, the European Unions Horizon 2020 Research and Innovation Program under Grant Agreement No. 820445 and Project Name Quantum Internet Alliance, Alberta Innovates Technology Futures, the National Sciences and Engineering Research Council of Canada, the Alberta Ministry of Jobs, Economy and Innovation's Major Innovation Fund Project on Quantum Technologies. Furthermore, W. T. acknowledges funding as a Senior Fellow of the Canadian Institute for Advanced Research (CIFAR). This material is based in part on research at Montana State University sponsored by Air Force Research Laboratory under Agreement No. FA8750-20-1-1004.

*These authors contributed equally to this work.

[1] H. J. Kimble, Nature (London) 453, 1023 (2008).

[2] S. Wehner, D. Elkouss, and R. Hanson, Science 362, eaam9288 (2018).

[3] H.-J. Briegel, W. Dür, J. I. Cirac, and P. Zoller, Phys. Rev. Lett. 81, 5932 (1998).

[4] L.-M. Duan, M. D. Lukin, J. I. Cirac, and P. Zoller, Nature (London) 414, 413 (2001).

[5] N. Sangouard, C. Simon, H. de Riedmatten, and N. Gisin, Rev. Mod. Phys. 83, 33 (2011).

[6] N. Sinclair, E. Saglamyurek, H. Mallahzadeh, J. A. Slater, M. George, R. Ricken, M. P. Hedges, D. Oblak, C. Simon, W. Sohler, and W. Tittel, Phys. Rev. Lett. 113, 053603 (2014).

[7] L. Jiang, J. M. Taylor, K. Nemoto, W. J. Munro, R. Van Meter, and M. D. Lukin, Phys. Rev. A 79, 032325 (2009).

[8] A. I. Lvovsky, B. C. Sanders, and W. Tittel, Nat. Photonics 3, 706 (2009).

[9] M. Fleischhauer and M. D. Lukin, Phys. Rev. Lett. 84, 5094 (2000).

[10] B. Kraus, W. Tittel, N. Gisin, M. Nilsson, S. Kröll, and J. I. Cirac, Phys. Rev. A 73, 020302(R) (2006).

[11] M. Afzelius, I. Usmani, A. Amari, B. Lauritzen, A. Walther, C. Simon, N. Sangouard, J. Minár, H. de Riedmatten, N. Gisin, and S. Kröll, Phys. Rev. Lett. 104, 040503 (2010).

[12] Z.-Q. Zhou, Y.-L. Hua, X. Liu, G. Chen, J.-S. Xu, Y.-J. Han, C.-F. Li, and G.-C. Guo, Phys. Rev. Lett. 115, 070502 (2015).

[13] K. F. Reim, J. Nunn, V. O. Lorenz, B. J. Sussman, K. C. Lee, N. K. Langford, D. Jaksch, and I. A. Walmsley, Nat. Photonics 4, 218 (2010).

[14] J.-P. Dou, A.-L. Yang, M.-Y. Du, D. Lao, J. Gao, L.-F. Qiao, H. Li, X.-L. Pang, Z. Feng, H. Tang, and X.-M. Jin, Commun. Phys. 1, 55 (2018).

[15] See Supplemental Material at http://link.aps.org/supplemental/ 10.1103/PhysRevLett.127.220502 for a detailed discussion of the memory deadtime and repeater-based entanglement distribution rate, a short summary of the AFC efficiency and its current limitations, and details of the single-photon storage experiment. 
[16] M. Afzelius, C. Simon, H. de Riedmatten, and N. Gisin, Phys. Rev. A 79, 052329 (2009).

[17] C. W. Thiel, N. Sinclair, W. Tittel, and R. L. Cone, Phys. Rev. B 90, 214301 (2014).

[18] C. W. Thiel, N. Sinclair, W. Tittel, and R. L. Cone, Phys. Rev. Lett. 113, 160501 (2014).

[19] T. Böttger, C. W. Thiel, R. L. Cone, and Y. Sun, Phys. Rev. B 79, 115104 (2009).

[20] R. W. Equall, Y. Sun, R. L. Cone, and R. M. Macfarlane, Phys. Rev. Lett. 72, 2179 (1994).

[21] M. Businger, A. Tiranov, K. T. Kaczmarek, S. Welinski, Z. Zhang, A. Ferrier, P. Goldner, and M. Afzelius, Phys. Rev. Lett. 124, 053606 (2020).

[22] P. Jobez, N. Timoney, C. Laplane, J. Etesse, A. Ferrier, P. Goldner, N. Gisin, and M. Afzelius, Phys. Rev. A 93, 032327 (2016).

[23] S. Guha, H. Krovi, C. A. Fuchs, Z. Dutton, J. A. Slater, C. Simon, and W. Tittel, Phys. Rev. A 92, 022357 (2015).

[24] H. Krovi, S. Guha, Z. Dutton, J. A. Slater, C. Simon, and W. Tittel, Appl. Phys. B 122, 52 (2016).

[25] G. Liu and B. Jacquier, Spectroscopic Properties of Rare Earths in Optical Materials (Springer Science \& Business Media, New York, 2006), Vol. 83.

[26] C. Thiel, T. Böttger, and R. Cone, J. Lumin. 131, 353 (2011).

[27] T. Zhong and P. Goldner, Nanophotonics 8, 2003 (2019).

[28] F. Bussieres, N. Sangouard, M. Afzelius, H. de Riedmatten, C. Simon, and W. Tittel, J. Mod. Opt. 60, 1519 (2013).

[29] K. Heshami, D. G. England, P. C. Humphreys, P. J. Bustard, V. M. Acosta, J. Nunn, and B. J. Sussman, J. Mod. Opt. 63, 2005 (2016).

[30] E. Saglamyurek, N. Sinclair, J. A. Slater, K. Heshami, D. Oblak, and W. Tittel, New J. Phys. 16, 065019 (2014).

[31] A. Kinos, D. Hunger, R. Kolesov, K. Mlmer, H. de Riedmatten, P. Goldner, A. Tallaire, L. Morvan, P. Berger, S. Welinski, K. Karrai, L. Rippe, S. Krll, and A. Walther, arXiv:2103.15743.

[32] Y. Ma, Y.-Z. Ma, Z.-Q. Zhou, C.-F. Li, and G.-C. Guo, Nat. Commun. 12, 2381 (2021).

[33] P. Jobez, I. Usmani, N. Timoney, C. Laplane, N. Gisin, and M. Afzelius, New J. Phys. 16, 083005 (2014).

[34] A. Seri, A. Lenhard, D. Rieländer, M. Gündoğan, P. M. Ledingham, M. Mazzera, and H. de Riedmatten, Phys. Rev. X 7, 021028 (2017).

[35] A. Seri, D. Lago-Rivera, A. Lenhard, G. Corrielli, R. Osellame, M. Mazzera, and H. de Riedmatten, Phys. Rev. Lett. 123, 080502 (2019).

[36] J. F. Dillon and L. R. Walker, Phys. Rev. 124, 1401 (1961).

[37] J. H. Davidson, P. J. T. Woodburn, A. D. Marsh, K. J. Olson, A. Olivera, A. Das, M. F. Askarani, W. Tittel, R. L. Cone, and C. W. Thiel, Phys. Rev. B 104, 134103 (2021).

[38] M. 1. G. Puigibert, M.F. Askarani, J.H. Davidson, V. B. Verma, M. D. Shaw, S. W. Nam, T. Lutz, G. C. Amaral, D. Oblak, and W. Tittel, Phys. Rev. Research 2, 013039 (2020).

[39] Y. Sun, C. W. Thiel, and R. L. Cone, Phys. Rev. B 85, 165106 (2012).

[40] E. Saglamyurek, N. Sinclair, J. Jin, J. A. Slater, D. Oblak, F. Bussieres, M. George, R. Ricken, W. Sohler, and W. Tittel, Nature (London) 469, 512 (2011).
[41] E. Saglamyurek, T. Lutz, L. Veissier, M. P. Hedges, C. W. Thiel, R. L. Cone, and W. Tittel, Phys. Rev. B 92, 241111(R) (2015).

[42] L. Veissier, C. W. Thiel, T. Lutz, P. E. Barclay, W. Tittel, and R. L. Cone, Phys. Rev. B 94, 205133 (2016).

[43] M. P. Hedges, J. J. Longdell, Y. Li, and M. J. Sellars, Nature (London) 465, 1052 (2010).

[44] B. Lauritzen, N. Timoney, N. Gisin, M. Afzelius, H. de Riedmatten, Y. Sun, R. M. Macfarlane, and R. L. Cone, Phys. Rev. B 85, 115111 (2012).

[45] G. Kowzan, D. Charczun, A. Cygan, R. S. Trawiński, D. Lisak, and P. Masłowski, Sci. Rep. 9, 8206 (2019).

[46] D. M. S. Johnson, J. M. Hogan, S. w. Chiow, and M. A. Kasevich, Opt. Lett. 35, 745 (2010).

[47] R. Houtz, C. Chan, and H. Müller, Opt. Express 17, 19235 (2009).

[48] M. Grimau Puigibert, G. H. Aguilar, Q. Zhou, F. Marsili, M. D. Shaw, V. B. Verma, S. W. Nam, D. Oblak, and W. Tittel, Phys. Rev. Lett. 119, 083601 (2017).

[49] P.-C. Wang, O. Pietx-Casas, M.F. Askarani, and G. C. do Amaral, J. Opt. Soc. Am. B 38, 1140 (2021).

[50] T. van Leent, M. Bock, R. Garthoff, K. Redeker, W. Zhang, T. Bauer, W. Rosenfeld, C. Becher, and H. Weinfurter, Phys. Rev. Lett. 124, 010510 (2020).

[51] Y. Hu, M. Yu, D. Zhu, N. Sinclair, A. Shams-Ansari, L. Shao, J. Holzgrafe, M. Zhang, and M. Loncar, in Conference on Lasers and Electro-Optics (Optical Society of America, Washington, D.C., 2020), p. STu4J.4.

[52] S. F. Preble, Q. Xu, and M. Lipson, Nat. Photonics 1, 293 (2007).

[53] M. Lauermann, C. Weimann, A. Knopf, W. Heni, R. Palmer, S. Koeber, D. L. Elder, W. Bogaerts, J. Leuthold, L. R. Dalton, C. Rembe, W. Freude, and C. Koos, Opt. Express 24, 11694 (2016).

[54] Y. Zhao, J. Zhang, J. Stuhler, G. Schuricht, F. Lison, Z. Lu, and L. Wang, Opt. Commun. 283, 4696 (2010).

[55] J. Liu, T. Liu, L. Chen, L. Zhang, G. Xu, D. Jiao, and S. Zhang, Opt. Laser Technol. 136, 106777 (2021).

[56] A. Louchet-Chauvet, R. Ahlefeldt, and T. Chanelire, Rev. Sci. Instrum. 90, 034901 (2019).

[57] Y. Sun, G. M. Wang, R. L. Cone, R. W. Equall, and M. J. M. Leask, Phys. Rev. B 62, 15443 (2000).

[58] C. W. Thiel, R. M. Macfarlane, Y. Sun, T. Bttger, N. Sinclair, W. Tittel, and R. L. Cone, Laser Phys. 24, 106002 (2014).

[59] R.-C. Tongning, T. Chanelière, J.-L. Le Gouët, and M. F. Pascual-Winter, J. Phys. Conf. Ser. 605, 012037 (2015).

[60] D. L. McAuslan, J. G. Bartholomew, M. J. Sellars, and J. J. Longdell, Phys. Rev. A 85, 032339 (2012).

[61] A. Ruskuc, J. M. Kindem, J. G. Bartholomew, J. Rochman, Y. Q. Huan, and A. Faraon, in OSA Quantum 2.0 Conference (Optical Society of America, Washington, D.C., 2020), p. QTh6A.7.

[62] A. Ortu, A. Tiranov, S. Welinski, F. Fröwis, N. Gisin, A. Ferrier, P. Goldner, and M. Afzelius, Nat. Mater. 17, 671 (2018).

[63] M. Afzelius and C. Simon, Phys. Rev. A 82, 022310 (2010).

[64] M. Sabooni, Q. Li, S. Kröll, and L. Rippe, Phys. Rev. Lett. 110, 133604 (2013).

[65] J. H. Davidson, P. Lefebvre, J. Zhang, D. Oblak, and W. Tittel, Phys. Rev. A 101, 042333 (2020). 
[66] M. Sabooni, S. T. Kometa, A. Thuresson, S. Kröll, and L. Rippe, New J. Phys. 15, 035025 (2013).

[67] M. Bonarota, J. Ruggiero, J. L. Le Gouët, and T. Chanelière, Phys. Rev. A 81, 033803 (2010).

[68] L. Rippe, M. Nilsson, S. Kröll, R. Klieber, and D. Suter, Phys. Rev. A 71, 062328 (2005).
[69] M. Bonarota, J.-L. Le Gouët, and T. Chanelière, New J. Phys. 13, 013013 (2011).

[70] M. H. Abobeih, J. Randall, C. E. Bradley, H. P. Bartling, M. A. Bakker, M. J. Degen, M. Markham, D. J. Twitchen, and T. H. Taminiau, Nature (London) 576, 411 (2019). 